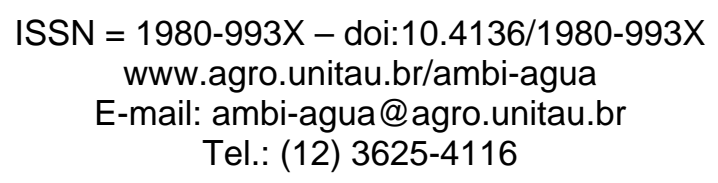

\title{
Crescimento da mamoneira sob diferentes tipos de águas residuárias e níveis de água no solo \\ (doi:10.4136/ambi-agua.112)
}

\author{
Josilda de França Xavier ${ }^{1}$; Carlos Alberto Vieira de Azevedo ${ }^{2}$; Napoleão Esberard de \\ Macêdo Beltrão ${ }^{3}$; Antonio Ricardo Santos de Andrade ${ }^{4}$; Vera Lúcia Antunes de Lima ${ }^{2}$ \\ ${ }^{1}$ Departamento de Agroecologia, Universidade Estadual da Paraíba, UEPB \\ E-mail: josilda.f.xavier@gmail.com \\ ${ }^{2}$ Departamento de Engenharia Agrícola, Universidade Federal de Campina Grande, UFCG \\ E-mail: cazevedo@deag.ufcg.edu.br; antuneslima@gmail.com \\ ${ }^{3}$ Centro Nacional de Pesquisa do Algodão, EMBRAPA \\ E-mail: napoleao@cnpa.embrapa.br \\ ${ }^{4}$ Universidade Federal Rural de Pernambuco, UFRPE \\ E-mail: arsauag@uag.ufrpe.br
}

\section{RESUMO}

Este trabalho avaliou o efeito de diferentes níveis de água no solo e tipos de águas residuárias tratadas provenientes de indústrias de Campina Grande, $\mathrm{PB}$, no crescimento da mamoneira BRS Nordestina. O experimento foi conduzido em casa de vegetação do Centro Nacional de Pesquisa do Algodão. O delineamento experimental foi em blocos inteiramente casualizados com 15 tratamentos em esquema de análise fatorial adicional [ ( 4 x 3) + 3] com três repetições, tendo os seguintes fatores: três tipos de águas residuárias tratadas e água de abastecimento $\left(\mathrm{A}_{1}=\right.$ IPELSA - Indústria de Celulose e Papel da Paraíba S/A; $A_{2}=$ COTEMINAS - Indústria de beneficiamento da fibra do algodão $\mathrm{S} / \mathrm{A} ; \mathrm{A}_{3}=$ ILCASA Indústria de laticínios de Campina Grande S/A (LEBOM); $\mathrm{A}_{4}=$ Água da rede de abastecimento público da cidade de Campina Grande, três níveis de água disponível no solo (AD) $\left(\mathrm{N}_{1}=100 \%, \mathrm{~N}_{2}=80 \%\right.$ e $\left.\mathrm{N}_{3}=70 \%\right)$ e três testemunhas, uma para cada AD com água de abastecimento e com fertilizante inorgânico na fundação ( $\left.\mathrm{A}_{4 \mathrm{C}}\right)$. Para avaliar o crescimento da mamoneira durante um período de 135 dias, foram realizadas medidas quinzenais das variáveis altura de planta, diâmetro do caule e área foliar total. Para todas as variáveis de crescimento houve interações significativas entre os fatores estudados, denotando a interdependência entre eles, o que foi refletido no crescimento das plantas. A mamoneira, cultivar BRS Nordestina, respondeu bem à irrigação com água residuária tratada, em especial da indústria COTEMINAS, principalmente quando associada ao nível de $100 \%$ da água disponível no solo.

Palavras-chave: irrigação; água residuária; biodiesel.

\section{Growth of castor bean plant under different types of wastewaters and soil water levels}

\section{ABSTRACT}

This work evaluated the effect of different levels of water into the soil and types of treated wastewaters from industries of Campina Grande city, Paraíba state, on the growth of castor bean plant, variety BRS Nordestina. The experiment was conducted in greenhouse of the National Center of Research of Cotton. The experimental design was in entirely randomized blocks with 15 treatments in scheme of additional factorial analysis [ $(4 \times 3)+3]$ with three replications, having the following factors: three types of treated wastewaters and 
water of provisioning $\left(A_{1}=\right.$ IPELSA - Industry of Cellulose and Paper of Paraíba S/A; $A_{2}=$ COTEMINAS - Industry of improvement of cotton fiber S/A; $A_{3}=$ ILCASA - Industry of dairy products of Grande S/A (LEBOM); $\mathrm{A}_{4}=$ Water of the network of public provisioning of Campina Grande city, three levels of available soil water (AW) $\left(\mathrm{N}_{1}=100 \%, \mathrm{~N}_{2}=80 \%\right.$ and $\mathrm{N}_{3}=70 \%$ ) and three controls, one for each AW with water of provisioning and with inorganic fertilizer in the foundation $\left(\mathrm{A}_{4} \mathrm{C}\right)$. In order to evaluate the growth of the castor bean plant during a period of 135 days, biweekly measures of the plant height, diameter of the stem and total foliar area variables were accomplished. For all growth variables there were significant interactions among the studied factors, denoting the interdependence among them, what was reflected on the growth of the plants. The castor bean plant, variety BRS Nordestina, responded well to irrigation with treated wastewater, especially from COTEMINAS industry mainly when associated to the level of $100 \%$ of the available soil water.

Keywords: irrigation; treated wastewater; biodiesel.

\section{INTRODUÇÃO}

A escassez dos recursos hídricos e o crescimento explosivo da população obrigam a priorização do uso das águas superficiais para o abastecimento público e a geração de energia elétrica, ficando, consequentemente, as atividades agrícolas comprometidas (Mancuso, 2003). De modo geral, a poluição das águas pode ocorrer, principalmente, por esgotos sanitários, águas residuárias industriais, lixiviação e percolação de fertilizantes e pesticidas, precipitação de efluentes atmosféricos e inadequada disposição dos resíduos sólidos (Studart e Campos, 2001).

O reúso das águas residuárias tratadas é considerado um excelente instrumento para otimização dos recursos hídricos, cada vez mais ameaçados de escassez. Mais que isso, é uma forma de desenvolvimento sustentável, podendo os recursos hídricos ser aproveitados de forma permanente. Outro fator importante também levado em consideração na reutilização das águas residuárias das indústrias, é a conscientização ambiental, que vem se destacando dia a dia, nos diversos setores da sociedade moderna, com uma cobrança cada vez maior da sociedade civil organizada às autoridades competentes, bem como aos setores produtivos da sociedade. Com efeito, as alterações que vêm ocorrendo no meio ambiente, sobretudo pelo descarte de resíduos industriais, de forma desordenada, vêm ocasionando a escassez de água de boa qualidade, reorientando o empresário a uma mudança de comportamento, no mundo inteiro, do ponto de vista técnico/ambiental, que minimize os impactos ambientais e preserve o ecossistema às gerações futuras (Sousa e Leite, 2003).

Segundo Von Sperling (1996), os esgotos industriais variam essencialmente com o tipo da indústria e com o processo industrial utilizado. Portanto, apresenta uma ampla variabilidade das suas características qualitativas, o que dificulta uma generalização dos valores mais comuns. Em termos do tratamento biológico dos esgotos industriais, assumem importância os seguintes aspectos: biodegradabilidade, tratabilidade, concentração de matéria orgânica, disponibilidade de nutrientes e toxidez.

Países desenvolvidos e em desenvolvimento vêm adotando consideravelmente a prática do reúso de água residuária tratada no cultivo de várias espécies, tais como leguminosas, forrageiras, gramíneas e hortaliças, principalmente nas regiões áridas e semiáridas, devido à água ter se tornado um fator limitante para o desenvolvimento urbano, industrial e agrícola. Nos últimos anos no Brasil, os estudos científicos sobre o reúso de águas residuárias tratadas na irrigação tiveram um papel importante, resultando em avanços tecnológicos na agricultura, a exemplo dos resultados de pesquisas apresentados no Workshop Uso e Reúso de Águas de Qualidade Inferior, realizado em Campina Grande, PB, em novembro de 2005. Nas regiões 
áridas e semiáridas do planeta, o reúso de efluentes de estação de tratamento de esgotos vem crescendo a cada dia, melhorando a qualidade de vida e as condições socioeconômicas das populações do meio rural, com o aumento da produtividade agrícola, recuperação de áreas degradadas ou improdutivas; e, ainda, no que diz respeito ao meio ambiente, contribuindo para a conservação e preservação dos recursos hídricos, evitando a descarga de esgoto bruto nos mananciais (Amorim Neto e Beltrão, 1999).

A cultura da mamona (Ricinus communis L), uma das 7.000 espécies da família das Euforbiáceas, é de elevada importância para o semiárido brasileiro por ser de fácil cultivo, resistente à seca, além de proporcionar ocupação e renda no meio rural, sendo bastante cultivada por pequenos produtores.

O óleo é o seu principal produto em importação econômica, único na natureza solúvel em álcool, com inúmeras aplicações industriais, como plásticos, fibras sintéticas, tintas e esmaltes, lubrificantes, entre outros (Beltrão et al., 2001). O uso do óleo na ricinoquímica ainda é pequeno, porém com ampla possibilidade de ser utilizado para a fabricação de biodiesel, com perspectiva de área plantada dez vez maior que a atual, podendo o Brasil passar a ser o maior produtor mundial. Além disso, recentemente, na Europa, foi proibido o uso do enxofre como agente aditivo de lubricidade do diesel mineral; dessa forma, o óleo da mamona poderá ser o seu substituto, pois tem cerca de 35\% a mais de lubricidade do que os demais óleos. Considerando ainda que o enxofre em contato com a água da atmosfera, forma acido sulfúrico, ocasionando a chuva ácida, um dos eventos mais poluidores do mundo moderno (Azevedo et al., 2001). Nos últimos três anos, tem-se intensificado o Programa Nacional de Biocombustível, e para a região Nordeste, a mamona tem sido considerada como uma das principais matérias-primas para fabricação do biodiesel.

O uso de águas residuárias no cultivo da mamona poderá reduzir o custo de produção de biodiesel e colaborar para a redução da poluição do ambiente, aspectos de suma importância no mundo atual, além de gerar trabalho e renda (Araújo et al., 2000). Diante da importância de se buscar novas fontes de água a serem utilizadas na agricultura, a presente pesquisa teve por objetivo avaliar os efeitos de níveis e tipos de águas residuárias tratadas provenientes de indústrias da cidade de Campina Grande-PB (IPELSA - Indústria de Celulose e Papel da Paraíba S/A; COTEMINAS - S/A; ILCASA - Indústria de Laticínios de Campina Grande S/A (LEBOM)) sobre o crescimento da mamoneira BRS Nordestina.

\section{MATERIAL E MÉTODOS}

O experimento foi conduzido, no período de 5 de maio a 6 de outubro de 2006, com a planta da mamoneira (Ricinus communis L.), cultivar BRS Nordestina, em condições de casa de vegetação do Centro Nacional de Pesquisa de Algodão (CNPA/EMBRAPA), localizado na cidade de Campina Grande, PB, Brasil, cujas coordenadas geográficas são as seguintes: $7^{\circ}$ 13' 11' de latitude sul, 35 52' 31'’de longitude oeste e a $550 \mathrm{~m}$ de altitude (IBGE, 2000). De acordo com a classificação climática de Kôppen adaptada ao Brasil (Coelho e Soncin, 1982), o clima é do tipo "CSa”, que é classificado como mesotérmico, semiúmido, com verão quente e seco, tendo uma duração de quatro a cinco meses e chuvas de outono e inverno. Também se observam climas frios nos meses de junho e julho com médias de temperaturas inferiores a $20^{\circ} \mathrm{C}$ (IBGE, 1991).

O delineamento experimental foi em blocos inteiramente casualizados contendo 15 tratamentos em esquema de análise fatorial adicional [( $4 \times 3)+3]$ com três repetições, tendo os seguintes fatores: três tipos de águas residuárias tratadas e água de abastecimento $\left(\mathrm{A}_{1}=\right.$ IPELSA; $\mathrm{A}_{2}=$ COTEMINAS; $\mathrm{A}_{3}=\mathrm{LEBOM} ; \mathrm{A}_{4}=$ Água da rede de abastecimento público da cidade de Campina Grande-PB), três níveis de água disponível no solo (AD) $\left(\mathrm{N}_{1}=100 \%, \mathrm{~N}_{2}\right.$ $=80 \%$ e $\mathrm{N}_{3}=70 \%$ da água disponível) e três testemunhas uma para cada nível de água 
XAVIER, J. F.; AZEVEDO, C. A. V.; BeltrÃO, N. E. M.; ANDRADE, A. R. S.; LiMA, V. L. A. Crescimento da mamoneira sob diferentes tipos de águas residuárias e níveis de água no solo. Ambi-Agua, Taubaté, v. 4, n. 3, p. 196-210, 2009. (doi:10.4136/ambi-agua.112)

disponível no solo (AD) com água de abastecimento e com fertilizante inorgânico na fundação $\left(\mathrm{A}_{4 \mathrm{C}}\right)$.

No experimento foram utilizadas sementes pré-selecionadas, da cultivar BRS Nordestina que possui uma grande resistência à seca; seu ciclo tem uma duração média de 150 dias, sua altura média é de 1,70 a 2,00 m de altura, seu fruto é semi-indescente, apresenta uma semente de bom calibre, que varia entre $68 \mathrm{~g}$ por 100 sementes, dando uma produtividade entre 1.200 a $1.500 \mathrm{~kg} \mathrm{ha}^{-1}$ em regime de sequeiro e de 3.500 a $4.000 \mathrm{~kg} \mathrm{ha}^{-1}$ em regime de irrigação, com o teor de óleo na semente acima de $47 \%$ (Beltrão et al., 2001).

O experimento se desenvolveu em vasos plásticos, de modo que cada unidade experimental correspondeu a um vaso com capacidade de 60 L com diâmetro inferior de 27 $\mathrm{cm}$, superior $41 \mathrm{~cm}$ e altura de $57 \mathrm{~cm}$; foram utilizados 45 vasos sob uma base de tijolo para facilitar a drenagem (Figura 1). Em cada vaso foi instalado um tensiômetro (Figura 1) a uma profundidade de $30 \mathrm{~cm}$ da superfície do solo, com o objetivo de se determinar o momento da irrigação e o volume de água a ser aplicado, o qual era calculado por uma planilha de Excel em função da leitura do tensiômetro.
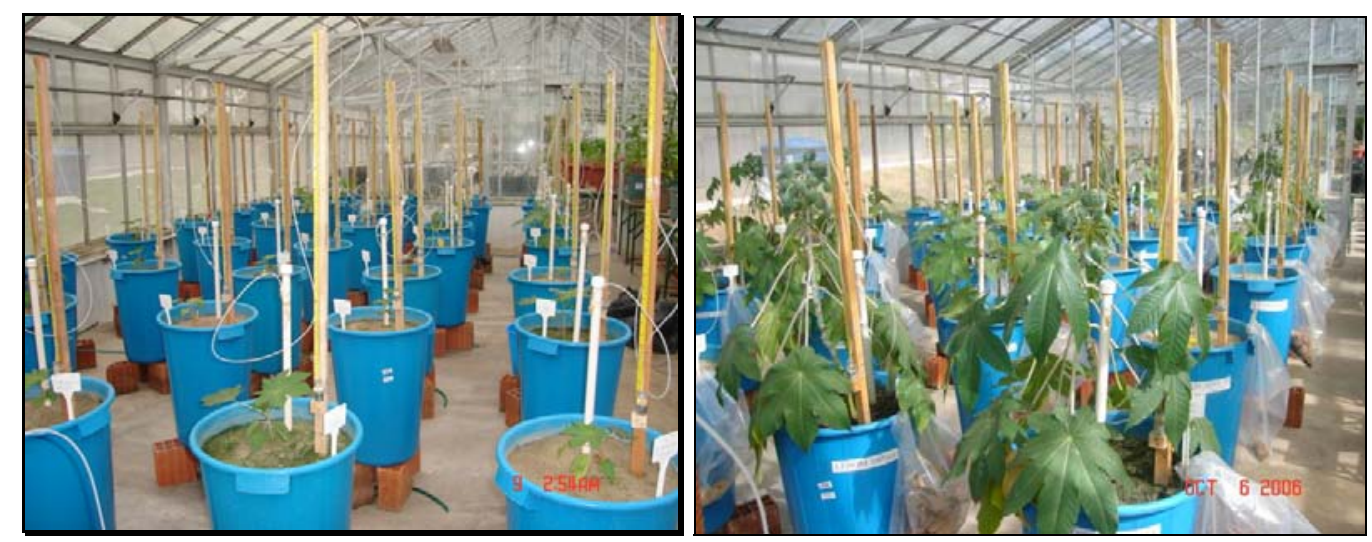

Figura 1. Visão geral no inicio e no final do experimento na casa de vegetação da Embrapa Algodão.

As Tabelas 1 a 3 apresentam as características químicas relativas aos macro e micronutrientes das águas residuárias das indústrias IPELSA, COTEMINAS e LEBOM utilizadas na pesquisa, cujas análises foram feitas no Laboratório de Irrigação e Salinidade (LIS/DEAg/CTRN/UFCG) Campina Grande-PB, no Laboratório da EXTRABES (Estação Experimental de Tratamento Biológico de Esgoto Sanitário) UFPB/UEPB e no Laboratório de Química e Fertilidade do Solo da Universidade Federal da Paraíba, Centro de Ciências Agrárias, Campus II, Areia-PB. 
XAVIER, J. F.; AZEVEDO, C. A. V.; BELTRÃO, N. E. M.; ANDRADE, A. R. S.; LIMA, V. L. A. Crescimento da mamoneira sob diferentes tipos de águas residuárias e níveis de água no solo. Ambi-Agua, Taubaté, v. 4, n. 3, p. 196-210, 2009. (doi:10.4136/ambi-agua.112)

Tabela 1. Análises dos macronutrientes das águas residuárias tratadas das indústrias IPELSA, COTEMINAS E LEBOM.

\begin{tabular}{|c|c|c|c|c|c|c|}
\hline Indústrias & Datas & $\begin{array}{c}\mathrm{Na} \\
\left(\mathrm{mg} \mathrm{L}^{-1}\right)\end{array}$ & $\begin{array}{c}\mathbf{K} \\
\left(\mathrm{mg} \mathrm{L}^{-1}\right)\end{array}$ & $\begin{array}{c}\mathbf{C a} \\
\left(\mathrm{mg} \mathrm{L}^{-1}\right)\end{array}$ & $\begin{array}{c}\mathrm{Mg} \\
\left(\mathrm{mg} \mathrm{L}^{-1}\right)\end{array}$ & $\begin{array}{l}\text { Fósforo } \\
\left(\mathrm{mg} \mathrm{L}^{-1}\right)\end{array}$ \\
\hline IPELSA & $12 / 06 / 2006$ & 29,44 & 24,99 & 93,25 & 95,25 & 2,67 \\
\hline IPELSA & $27 / 06 / 2006$ & 211,41 & 25,71 & 191,25 & 34,2 & 0,39 \\
\hline IPELSA & $12 / 07 / 2006$ & 211,41 & 24,27 & 168,25 & 46,65 & 1,73 \\
\hline IPELSA & 27/07/2006 & 211,41 & 25,71 & 246,50 & 65,7 & 2,05 \\
\hline IPELSA & $11 / 08 / 2006$ & 211,37 & 24,96 & 234,60 & 21,84 & 1,52 \\
\hline IPELSA & $26 / 08 / 2006$ & 29,21 & 26,13 & 165,40 & 58,8 & * \\
\hline IPELSA & $10 / 09 / 2006$ & 317,17 & 26,13 & 196,60 & 56,52 & $*$ \\
\hline COTEMINAS & $12 / 06 / 2006$ & 124,66 & 28,58 & 19,00 & 21,45 & 8,58 \\
\hline COTEMINAS & $27 / 06 / 2006$ & 131,01 & 28,58 & 18,50 & 26,85 & 6,5 \\
\hline COTEMINAS & $12 / 07 / 2006$ & 128,89 & 25,71 & 20,00 & 21,6 & 8,9 \\
\hline COTEMINAS & 27/07/2006 & 135,24 & 27,14 & 24,50 & 24,3 & 7,45 \\
\hline COTEMINAS & $11 / 08 / 2006$ & 145,59 & 28,47 & 22,00 & 26,04 & 9,33 \\
\hline COTEMINAS & $26 / 08 / 2006$ & 141,45 & 31,20 & 31,00 & 18,96 & * \\
\hline COTEMINAS & $10 / 09 / 2006$ & 143,52 & 31,98 & 18,20 & 29,40 & * \\
\hline LEBOM & $12 / 06 / 2006$ & 126,77 & 26,43 & 46,50 & 37,65 & 8,14 \\
\hline LEBOM & $27 / 06 / 2006$ & 135,24 & 21,40 & 30,00 & 28,95 & 5,99 \\
\hline LEBOM & $12 / 07 / 2006$ & 82,34 & 14,23 & 27,25 & 33,75 & 9,05 \\
\hline LEBOM & $27 / 07 / 2006$ & 145,82 & 7,77 & 20,50 & 8,40 & 4,22 \\
\hline LEBOM & $11 / 08 / 2006$ & 211,37 & 20,67 & 30,60 & 14,64 & 7,69 \\
\hline LEBOM & $26 / 08 / 2006$ & 75,90 & 24,18 & 30,00 & 16,56 & $*$ \\
\hline LEBOM & $10 / 09 / 2006$ & 147,89 & 12,48 & 31,00 & 25,20 & * \\
\hline
\end{tabular}

Nota: Análises realizadas no Laboratório de Irrigação e Salinidade (LIS/DEAg/CTRN/UFCG) Campina Grande-PB.

Tabela 2. Análises dos macronutrientes das águas residuárias tratadas das Indústrias IPELSA, COTEMINAS e LEBOM.

\begin{tabular}{|c|c|c|c|c|}
\hline Indústrias & Datas & $\begin{array}{c}\text { Nitrogênio } \\
\text { Amoniacal } \\
\left(\mathrm{mg} \mathrm{L}^{-1}\right)\end{array}$ & $\begin{array}{c}\text { Nitrito } \\
\left(\mathrm{mg} \mathrm{L}^{-1}\right)\end{array}$ & $\begin{array}{l}\text { Nitrato } \\
\left(\mathrm{mg} \mathrm{L}^{-1}\right)\end{array}$ \\
\hline IPELSA & $27 / 07 / 2006$ & 15,95 & 11,69 & 3,03 \\
\hline IPELSA & $11 / 08 / 2006$ & 10,30 & 30,71 & 2,22 \\
\hline IPELSA & $26 / 08 / 2006$ & 6,01 & 211,50 & 0,42 \\
\hline IPELSA & $10 / 09 / 2006$ & 7,71 & 102,80 & 1,80 \\
\hline COTEMINAS & $27 / 07 / 2006$ & 48,47 & 27,22 & 2,38 \\
\hline COTEMINAS & $11 / 08 / 2006$ & 37,29 & 54,27 & 0,91 \\
\hline COTEMINAS & $26 / 08 / 2006$ & 32,55 & 766,07 & 0,83 \\
\hline COTEMINAS & $10 / 09 / 2006$ & 40,22 & 217,44 & 1,78 \\
\hline LEBOM & $27 / 07 / 2006$ & 28,26 & $*$ & 0,30 \\
\hline LEBOM & $11 / 08 / 2006$ & 27,92 & 68,32 & 8,67 \\
\hline LEBOM & 26/08/2006 & 13,13 & 195,26 & 0,95 \\
\hline LEBOM & $10 / 09 / 2006$ & 30,69 & 165,53 & 0,45 \\
\hline
\end{tabular}

Nota: As análises foram realizadas no laboratório da EXTRABES (Estação Experimental de Tratamento Biológica de Esgoto Sanitário) UFPB/UEPB. Metodologia (Silva e Oliveira, 2001) Campina Grande, PB, 2006; DBO ${ }^{1}$ - Demanda Bioquímica de Oxigênio e DQO ${ }^{2}$ - Demanda Química de Oxigênio. *não houve leitura 
XAVIER, J. F.; AZEVEDO, C. A. V.; BELTRÃO, N. E. M.; ANDRADE, A. R. S.; LIMA, V. L. A. Crescimento da mamoneira sob diferentes tipos de águas residuárias e níveis de água no solo. Ambi-Agua, Taubaté, v. 4, n. 3, p. 196-210, 2009. (doi:10.4136/ambi-agua.112)

Tabela 3. Análises dos micronutrientes das águas residuárias tratadas das indústrias IPELSA, COTEMINAS e LEBOM.

\begin{tabular}{|c|c|c|c|c|c|}
\hline Indústrias & Datas & $\begin{array}{c}\mathrm{Cu} \\
\left(\mathrm{mg} \mathrm{L}^{-1}\right)\end{array}$ & $\begin{array}{c}\mathrm{Mn} \\
\left(\mathrm{mg} \mathrm{L}^{-1}\right)\end{array}$ & $\begin{array}{c}\mathrm{Zn} \\
\left(\mathrm{mg} \mathrm{L}^{-1}\right)\end{array}$ & $\begin{array}{c}\text { Fe } \\
\left(\mathrm{mg} \mathrm{L}^{-1}\right)\end{array}$ \\
\hline IPELSA & 09/05/2006 & 0,04 & $<\mathrm{LD}^{1}$ & 0,20 & 2,69 \\
\hline IPELSA & 22/06/2006 & $*$ & 0,81 & 0,06 & 2,37 \\
\hline IPELSA & 13/07/2006 & $*$ & 0,69 & 0,07 & 1,66 \\
\hline COTEMINAS & 09/05/2006 & 0,03 & $<\mathrm{LD}^{1}$ & 0,15 & 2,37 \\
\hline COTEMINAS & 22/06/2006 & $*$ & 0,22 & 0,02 & 1,66 \\
\hline COTEMINAS & 13/07/2006 & $*$ & 0,14 & 0,03 & 1,58 \\
\hline LEBOM & 09/05/2006 & 0,13 & $<\mathrm{LD}^{1}$ & 0,24 & 5,08 \\
\hline LEBOM & 22/06/2006 & $*$ & 0,14 & 0,06 & 5,16 \\
\hline LEBOM & 13/07/2006 & $*$ & $<\mathrm{LD}^{1}$ & 0,04 & 5,71 \\
\hline
\end{tabular}

Nota: As análises foram realizadas no laboratório de Química e Fertilidade do Solo da Universidade Federal da Paraíba, Centro de Ciências Agrárias, Campus II - Areia - PB; $<\mathrm{LD}^{1}$ : Abaixo do Limite de Detecção do aparelho - 0,01 $\mathrm{mg} \mathrm{L}^{-1} ;<\mathrm{LD}^{2}$ : Abaixo do Limite de Detecção do aparelho-0,05 mg L $\mathrm{L}^{-1 ;}<\mathrm{LD}^{3}$ : Abaixo do Limite de Detecção do aparelho- $0,1 \mu \mathrm{L}^{-1}$; *limite de detecção menor que $0.001 \mathrm{mg} \mathrm{L}^{-1}$.

As análises físicas e químicas do solo encontram-se nas Tabelas 4 e 5 e foram realizadas no Laboratório de Irrigação e Salinidade (LIS) do Departamento de Engenharia Agrícola da Universidade Federal de Campina Grande-PB, utilizando a metodologia da EMBRAPA (1997).

Tabela 4. Características físicas do solo antes da instalação do experimento.

\begin{tabular}{|c|c|c|c|c|c|c|c|c|c|}
\hline \multicolumn{2}{|c|}{$\begin{array}{c}\text { Densidade } \\
\left(\mathrm{g} \mathrm{cm}^{3}\right)\end{array}$} & \multicolumn{3}{|c|}{$\begin{array}{c}\text { Granulométrica } \\
\mathrm{g} \mathrm{kg}^{-1}\end{array}$} & Textura & $\begin{array}{l}\text { CC } \\
(\%)\end{array}$ & $\begin{array}{c}\text { PMP } \\
(\%)\end{array}$ & $\begin{array}{l}\text { PT } \\
(\%)\end{array}$ & $\begin{array}{l}\text { AD } \\
(\%)\end{array}$ \\
\hline $\begin{array}{l}\text { Global } \\
1,65\end{array}$ & $\begin{array}{l}\text { Real } \\
2,57\end{array}$ & $\begin{array}{l}\text { Areia } \\
753,5\end{array}$ & $\begin{array}{l}\text { Silte } \\
84,2\end{array}$ & $\begin{array}{c}\text { Argila } \\
162,3\end{array}$ & Franco arenoso & 2,85 & 2,04 & 37,74 & 0,81 \\
\hline
\end{tabular}

Nota: Análises realizadas no Laboratório de Irrigação e Salinidade (LIS/DEAg/CTRN/UFCG) Campina GrandePB 2006. CC - Capacidade de campo; PMP - Ponto de murcha permanente; PT - Porosidade total; AD - água disponível.

Tabela 5. Características químicas (fertilidade) do solo antes da instalação do experimento.

\begin{tabular}{|c|c|c|c|c|c|c|c|c|c|c|c|}
\hline $\begin{array}{c}\mathbf{p H} \\
\mathrm{H}_{2} \mathrm{O}\end{array}$ & $\begin{array}{c}\mathrm{CE} \\
\left(\mathrm{Ds} \mathrm{m}^{-1}\right)\end{array}$ & \multicolumn{8}{|c|}{ Complexo Sortivo $\left(\mathrm{cmol}_{\mathrm{C}} \mathrm{kg}^{-1}\right)$} & $\left(\mathrm{mg} \mathrm{dm} \mathbf{m}^{-3}\right)$ & $\left(\mathrm{g} \mathrm{kg}^{-1}\right)$ \\
\hline $1: 2,5$ & CE & $\mathrm{Ca}^{+2}$ & $\mathrm{Mg}^{+2}$ & $\mathrm{Na}^{+}$ & $\mathrm{K}^{+}$ & S & $\mathrm{H}$ & $\mathrm{Al}$ & $\mathrm{T}$ & $\mathrm{P}$ & MO \\
\hline 5,53 & 0,14 & 0,43 & 0,17 & 0,04 & 0,09 & 0,73 & 0,18 & 0,30 & 1,21 & 0,40 & 10,9 \\
\hline
\end{tabular}

Nota: Análises realizadas no Laboratório de Irrigação e Salinidade (LIS) do DEAg/CTRN/UFCG Campina GrandePB 2006. MO - matéria orgânica; S - Soma de bases trocáveis do solo e T - S+ H +Al - Total da Soma de bases trocáveis do solo Hidrogênio mais Alumínio.

A adubação foi realizada com base nos resultados da análise química do solo (Embrapa, 1997) e na necessidade nutricional da planta; utilizaram-se no experimento três fontes de adubo químico: sultato de amônio $(20 \% \mathrm{~N})$; superfosfato triplo $\left(43 \% \mathrm{P}_{2} \mathrm{O}_{5}\right)$ e cloreto de potássio $\left(60 \% \mathrm{~K}_{2} \mathrm{O}\right)$, com o objetivo de elevar a fertilidade do solo. Foi realizada uma adubação de fundação no dia 27/04/06 com as seguintes doses: nitrogênio 2,2 $\mathrm{g}_{\text {vaso }}{ }^{-1}$, fósforo $2,76 \mathrm{~g} \mathrm{vaso}^{-1}$ e potássio $1,76 \mathrm{~g}$ vaso $^{-1}$.

Durante o período de 135 dias foi feita a avaliação das características de crescimento da mamoneira BRS Nordestina, em que a cada 15 dias foram realizadas medidas da altura da planta (AP), diâmetro do caule (DC) e área foliar total (AFT). O efeito dos fatores (tipos de 
XAVIER, J. F.; AZEVEDO, C. A. V.; BELTRÃO, N. E. M.; ANDRADE, A. R. S.; LIMA, V. L. A. Crescimento da mamoneira sob diferentes tipos de águas residuárias e níveis de água no solo. Ambi-Agua, Taubaté, v. 4, n. 3, p. 196-210, 2009. (doi:10.4136/ambi-agua.112)

águas residuárias e níveis de água disponível no solo) sobre o crescimento da mamoneira BRS Nordestina foi avaliado aplicando análise de variância simples e teste " $F$ ", segundo Santos et al. (2008).

Os resultados com respeito ao volume de água de irrigação foram também submetidos à análise de regressão, seguida de ajuste de função polinomial, em virtude da natureza quantitativa dos tratamentos. A escolha da equação de melhor ajuste foi baseada na significância do efeito da regressão, dos desvios testados pelo teste $\mathrm{F}$ a $5 \%$ e no maior coeficiente de determinação $\left(\mathrm{R}^{2}\right)$. Os coeficientes das equações de regressão foram testados até $10 \%$ pelo teste “t” (Bussab e Morettin, 2003).

\section{RESULTADOS E DISCUSSÃO}

Os resultados das análises de variância referentes à altura de planta (AP), diâmetro do caule (DC) e área foliar total (AFT) da mamoneira para o período de 15 a 135 dias após semeadura - DAS são mostrados nas Tabelas 6 a 8 . Verificam-se, pelo teste F, diferenças significativas a 1 e $5 \%$ de probabilidade para os fatores água residuária (A) e níveis de água disponível no solo $(\mathrm{N})$ em relação às três variáveis analisadas. A interação $\mathrm{D}$ x $\mathrm{C}$ também foi significativa ao nível de 1 e $5 \%$ de probabilidade para as três variáveis de crescimento da mamoneira, indicando haver dependência entre os efeitos dos fatores tipos de águas residuárias (A) e níveis de água disponível no solo (B). Pode-se notar pelo teste F a existência de diferenças significativas entre os tratamentos e a testemunha (fator adicional) para as variáveis AP, DC e AFT.

Tabela 6. Análise de variância da variável altura da planta $(\mathrm{cm})$ ao longo do cultivo da mamoneira submetida aos diferentes tratamentos.

\begin{tabular}{|c|c|c|c|c|c|c|c|}
\hline \multirow[t]{2}{*}{ Fontes de Variação } & \multirow[t]{2}{*}{ GL } & \multicolumn{6}{|c|}{$\begin{array}{c}\text { Quadrado médio (QM) } \\
\text { Dias Após a Semeadura - DAS }\end{array}$} \\
\hline & & $15^{(1)}$ & 45 & 60 & 75 & 120 & 135 \\
\hline Tipos de água (A) & 3 & $1,89 \mathrm{~ns}$ & $\begin{array}{r}448,56 \\
* *\end{array}$ & $\begin{array}{r}1105,21 \\
* *\end{array}$ & $\begin{array}{r}1267,21 \\
* *\end{array}$ & $\begin{array}{r}2207,66 \\
* *\end{array}$ & $\begin{array}{r}1820,82 \\
* *\end{array}$ \\
\hline Níveis de água (N) & 2 & 4,94 ns & $98,89 * *$ & $325,99 * *$ & $363,44 * *$ & $600,00 * *$ & $330,77 * *$ \\
\hline$A \times N$ & 6 & $13,98 * *$ & $41,41 * *$ & $102,53 * *$ & $57,52 * *$ & $71,02 * *$ & $46,17 \mathrm{~ns}$ \\
\hline $\begin{array}{l}\text { Fatorial } \mathrm{x} \\
\text { Testemunha }\end{array}$ & 3 & $\begin{array}{r}165,647 \\
* *\end{array}$ & $50,25 * *$ & $50,16 * *$ & $82,26 * *$ & $66,76 * *$ & $44,77 \mathrm{~ns}$ \\
\hline Tratamentos & 14 & $42,60 \mathrm{~ns}$ & $\begin{array}{r}138,76 \\
* *\end{array}$ & $338,08 * *$ & $365,74 * *$ & $603,53 * *$ & $466,81 * *$ \\
\hline Blocos & 2 & $4,41 \mathrm{~ns}$ & $5,24 \mathrm{~ns}$ & $5,79 \mathrm{~ns}$ & $6,10 \mathrm{~ns}$ & $2,07 \mathrm{~ns}$ & $29,60 \mathrm{~ns}$ \\
\hline Resíduo & 28 & 3,57 & 10,14 & 7,99 & 8,97 & 5,78 & 42,69 \\
\hline Total corrigido & 44 & & & & & & \\
\hline CV (\%) & & 15,30 & 16,16 & 11,92 & 11,77 & 7,55 & 21,18 \\
\hline Média geral (cm) & & 12,34 & 19,70 & 23,72 & 25,45 & 31,84 & 30,85 \\
\hline
\end{tabular}

Nota: GL - grau de liberdade; ns - não significativo, * - significativo ao nível de 5\% de probabilidade pelo teste F, ** - significativo ao nível de $1 \%$ de probabilidade pelo teste F. CV= coeficiente de variância; ${ }^{(1)} 15$ DAS água de abastecimento sem fertilizante. 
XAVIER, J. F.; AZEVEDO, C. A. V.; BELTRÃO, N. E. M.; ANDRADE, A. R. S.; LIMA, V. L. A. Crescimento da mamoneira sob diferentes tipos de águas residuárias e níveis de água no solo. Ambi-Agua, Taubaté, v. 4, n. 3, p. 196-210, 2009. (doi:10.4136/ambi-agua.112)

Tabela 7. Análise de variância da variável diâmetro caulinar $(\mathrm{cm})$ ao longo do cultivo da mamoneira submetida aos diferentes tratamentos.

\begin{tabular}{|c|c|c|c|c|c|c|c|}
\hline \multirow[t]{2}{*}{ Fontes de Variação } & \multirow[t]{2}{*}{ GL } & \multicolumn{6}{|c|}{$\begin{array}{l}\text { Quadrado médio (QM) } \\
\text { Dias Após a Semeadura }\end{array}$} \\
\hline & & $15^{(1)}$ & 45 & 60 & 75 & 120 & 135 \\
\hline Tipos de Água (A) & 3 & $0,00004 \mathrm{~ns}$ & $469,98 * *$ & $596,85 * *$ & $592,63 * *$ & $730,87 * *$ & $926,71 * *$ \\
\hline Níveis de água (N) & 2 & 0,000003 ns & $45,66 * *$ & $212,23 * *$ & $99,11 * *$ & $109,13 * *$ & $292,46 * *$ \\
\hline $\mathrm{A} \times \mathrm{N}$ & 6 & 0,00004 ns & $16,80 * *$ & $45,56 * *$ & $26,25 \mathrm{~ns}$ & $14,43 * *$ & $82,75 \mathrm{~ns}$ \\
\hline Fatorial x Testemunha & 3 & $0,0013 *$ & $42,83 * *$ & $16,44 * *$ & $45,54 *$ & $1,55 * *$ & 17,04 ns \\
\hline Tratamentos & 14 & $0,0003 * *$ & $123,61 * *$ & $181,26 * *$ & $162,16 * *$ & $178,72 * *$ & $279,47 * *$ \\
\hline Blocos & 2 & $0,00004 \mathrm{~ns}$ & $12,50 \mathrm{~ns}$ & $1,97 \mathrm{~ns}$ & $27,78 \mathrm{~ns}$ & $1,02 \mathrm{~ns}$ & 29,16 ns \\
\hline Resíduo & 28 & 0,00005 & 3,89 & 2,34 & 14,89 & 3,20 & 36,90 \\
\hline Total corrigido & 44 & & & & & & 5004,24 \\
\hline CV (\%) & & 12,36 & 28,76 & 17,30 & 42,81 & 14,95 & 50,97 \\
\hline Média geral (cm) & & 0,058 & 6,87 & 8,83 & $592,63 * *$ & 11,97 & 11,92 \\
\hline
\end{tabular}

Nota: GL - grau de liberdade; ns - não significativo, * - significativo ao nível de 5\% de probabilidade pelo teste F, ** significativo ao nível de $1 \%$ de probabilidade pelo teste F. CV= coeficiente de variância; ${ }^{(1)} 15$ DAS água de abastecimento sem fertilizante.

Tabela 8. Análise de variância da variável Área foliar total $\left(\mathrm{cm}^{2}\right)$ ao longo do cultivo da mamoneira submetida aos diferentes tratamentos.

\begin{tabular}{|c|c|c|c|c|c|c|c|}
\hline \multirow[t]{2}{*}{$\begin{array}{c}\text { Fontes } \\
\text { de } \\
\text { Variação } \\
\end{array}$} & \multirow[t]{2}{*}{ GL } & \multicolumn{6}{|c|}{$\begin{array}{l}\text { Quadrado médio (QM) } \\
\text { Dias Após a Semeadura }\end{array}$} \\
\hline & & $15^{(1)}$ & 45 & 60 & 75 & 120 & 135 \\
\hline $\begin{array}{l}\text { Tipos de } \\
\text { Água (A) }\end{array}$ & 3 & $80.233,73 * *$ & $36.935 .954,03 * *$ & $40.318 .688,23 * *$ & $50.109 .756,64 * *$ & $83.872 .552,24 * *$ & $64.234 .865,28 * *$ \\
\hline $\begin{array}{l}\text { Níveis de } \\
\text { água }(\mathrm{N})\end{array}$ & 2 & $2.902,78 \mathrm{~ns}$ & $14.150 .537,00 * *$ & $17.563 .789,60 * *$ & $18.882 .585,70 * *$ & $11.656 .597,27 * *$ & $6.784 .444,09 * *$ \\
\hline$A \times N$ & 6 & $2.321,41 \mathrm{~ns}$ & $4.930 .530,13 * *$ & $3.759 .680,14 * *$ & $3.850 .068,91 * *$ & $2.767 .285,34 * *$ & $1.546 .206,03$ * \\
\hline $\begin{array}{l}\text { Fatorial } \\
\text { x Test. }\end{array}$ & 3 & $101.256,07 * *$ & $4.088 .943,755 * *$ & $7.741 .267,44 * *$ & $10.144 .847,54 * *$ & $15.239 .838,78 * *$ & $11.894 .889,02 * *$ \\
\hline Tratamentos & 14 & $40.300,21 * *$ & $12.925 .639,15 * *$ & $14.418 .966,22 * *$ & $17.259 .242,59 * *$ & $24.089 .577,12 * *$ & $17.945 .384,81 *$ \\
\hline Blocos & 2 & $4.389,24 \mathrm{~ns}$ & $180.355,35 \mathrm{~ns}$ & $465.580,51 \mathrm{~ns}$ & $732.890,96 \mathrm{~ns}$ & $727.628,42$ * & $1.062 .089,89 \mathrm{~ns}$ \\
\hline Resíduo & 28 & $4.024,00$ & $378.276,49$ & 428.124,93 & $747.652,54$ & $172.831,31$ & $460.603,89$ \\
\hline $\begin{array}{l}\text { Total } \\
\text { corrigido }\end{array}$ & 44 & & & & & & \\
\hline CV (\%) & & 35,46 & 37,80 & 36,28 & 43,51 & 18,19 & 32,48 \\
\hline $\begin{array}{l}\text { Média geral } \\
(\mathrm{cm})\end{array}$ & & 178,88 & $1.627,24$ & $1.803,58$ & $1.987,46$ & $2.285,99$ & $2.089,36$ \\
\hline
\end{tabular}

Nota: GL - grau de liberdade; ns - não significativo, * - significativo ao nível de 5\% de probabilidade pelo teste F, ** significativo ao nível de $1 \%$ de probabilidade pelo teste F. CV= coeficiente de variância; ${ }^{(1)} 15$ DAS água de abastecimento sem fertilizante.

Tem-se nas Tabelas 9 e 10 os valores médios da altura da planta (AP), diâmetro do caule (DC) e área foliar total (AFT), da mamoneira quando submetida aos fatores tipos de águas residuárias (A) e níveis de água disponível no solo (N) aos 15, 45, 60, 75, 120 e 135 dias após a semeadura (DAS). 
XAVIER, J. F.; AZEVEDO, C. A. V.; BeltrÃO, N. E. M.; ANDRADE, A. R. S.; LiMA, V. L. A. Crescimento da mamoneira sob diferentes tipos de águas residuárias e níveis de água no solo. Ambi-Agua, Taubaté, v. 4, n. 3, p. 196-210, 2009. (doi:10.4136/ambi-agua.112)

Tabela 9. Valores médios dos dados de altura da planta (AP), diâmetro caulinar (DC) e área foliar total (AFT) da mamoneira, submetida aos diferentes tipos de águas residuárias e às testemunhas.

Tipos de águas residuárias

$$
\text { Variáveis de crescimento }
$$

AP (cm) DC (cm) AFT $\left(\mathrm{cm}^{2}\right)$

15 Dias Após Semeadura

\begin{tabular}{lllr}
\hline $\mathrm{A}_{1}=$ IPELSA & $10,59 \mathrm{a}$ & $0,055 \mathrm{a}$ & $104,93 \mathrm{a}$ \\
$\mathrm{A}_{2}=$ COTEMINAS & $11,43 \mathrm{a}$ & $0,055 \mathrm{a}$ & $277,83 \mathrm{~b}$ \\
$\mathrm{~A}_{3}=$ LEBOM & $10,47 \mathrm{a}$ & $0,051 \mathrm{a}$ & $96,93 \mathrm{a}$ \\
$\mathrm{A}_{4}=$ ÁGUA DE & $10,51 \mathrm{a}$ & $0,052 \mathrm{a}$ & $71,58 \mathrm{a}$ \\
ABASTECIMENTO & & & \\
\hline
\end{tabular}

\begin{tabular}{lcrr}
\hline \multicolumn{4}{c}{ 45 Dias Após Semeadura } \\
\hline $\mathrm{A}_{1}=$ IPELSA & $15,02 \mathrm{a}$ & $0,70 \mathrm{a}$ & $654,78 \mathrm{a}$ \\
$\mathrm{A}_{2}=$ COTEMINAS & $28,34 \mathrm{c}$ & $15,55 \mathrm{c}$ & $4.678,36 \mathrm{c}$ \\
$\mathrm{A}_{3}=$ LEBOM & $19,96 \mathrm{~b}$ & $8,96 \mathrm{~b}$ & $2.055,54 \mathrm{~b}$ \\
$\mathrm{~A}_{4}=$ ÁGUA DE & $12,25 \mathrm{a}$ & $0,59 \mathrm{a}$ & $162,62 \mathrm{a}$ \\
ABASTECIMENTO & & & \\
\hline
\end{tabular}

\begin{tabular}{lcrr}
\hline \multicolumn{4}{c}{ 60 Dias Após Semeadura } \\
\hline $\mathrm{A}_{1}=$ IPELSA & $17,89 \mathrm{~b}$ & $4,20 \mathrm{~b}$ & $857,97 \mathrm{a}$ \\
$\mathrm{A}_{2}=$ COTEMINAS & $37,78 \mathrm{~d}$ & $19,33 \mathrm{~d}$ & $4.886,57 \mathrm{c}$ \\
$\mathrm{A}_{3}=$ LEBOM & $25,00 \mathrm{c}$ & $9,28 \mathrm{c}$ & $2.740,00 \mathrm{~b}$ \\
$\mathrm{~A}_{4}=$ ÁGUA DE & $12,04 \mathrm{a}$ & $0,59 \mathrm{a}$ & $166,57 \mathrm{a}$ \\
ABASTECIMENTO & & & \\
\hline
\end{tabular}

\begin{tabular}{lcrr}
\hline \multicolumn{4}{c}{ 75 Dias Após Semeadura } \\
\hline $\mathrm{A}_{1}=$ IPELSA & $18,66 \mathrm{~b}$ & $5,21 \mathrm{a}$ & $958,62 \mathrm{a}$ \\
$\mathrm{A}_{2}=$ COTEMINAS & $40,44 \mathrm{~d}$ & $19,22 \mathrm{c}$ & $5.415,29 \mathrm{c}$ \\
$\mathrm{A}_{3}=$ LEBOM & $28,22 \mathrm{c}$ & $11,89 \mathrm{~b}$ & $3.073,92 \mathrm{~b}$ \\
$\mathrm{~A}_{4}=$ ÁGUA DE & $13,44 \mathrm{a}$ & $0,60 \mathrm{a}$ & $146,21 \mathrm{a}$ \\
ABASTECIMENTO & & &
\end{tabular}

\begin{tabular}{lcrr}
\hline \multicolumn{4}{c}{ 120 Dias Após Semeadura } \\
\hline $\mathrm{A}_{1}=$ IPELSA & $23,88 \mathrm{~b}$ & $9,39 \mathrm{~b}$ & $748,48 \mathrm{~b}$ \\
$\mathrm{~A}_{2}=$ COTEMINAS & $49,77 \mathrm{~d}$ & $20,89 \mathrm{~d}$ & $6.824,75 \mathrm{~d}$ \\
$\mathrm{~A}_{3}=$ LEBOM & $41,44 \mathrm{c}$ & $17,44 \mathrm{c}$ & $3.455,19 \mathrm{c}$ \\
$\mathrm{A}_{4}=$ ÁGUA DE & $15,66 \mathrm{a}$ & $0,67 \mathrm{a}$ & $131,42 \mathrm{a}$ \\
ABASTECIMENTO & & \\
\hline \multicolumn{4}{c}{} \\
\hline $\mathrm{A}_{1}=$ IPELSA & 135 Dias Após Semeadura \\
$\mathrm{A}_{2}=$ COTEMINAS & $23,94 \mathrm{a}$ & $7,38 \mathrm{a}$ & $756,03 \mathrm{a}$ \\
$\mathrm{A}_{3}=$ LEBOM & $45,55 \mathrm{~b}$ & $23,33 \mathrm{~b}$ & $6.004,47 \mathrm{c}$ \\
$\mathrm{A}_{4}=$ ÁGUA DE & $41,22 \mathrm{~b}$ & $17,56 \mathrm{~b}$ & $3.234,40 \mathrm{~b}$ \\
ABASTECIMENTO & $15,44 \mathrm{a}$ & $0,66 \mathrm{a}$ & $143,23 \mathrm{a}$ \\
\hline
\end{tabular}

Nota: As médias seguidas da mesma letra na coluna não diferem estatisticamente ao nível de 1 e $5 \%$ de probabilidade no Teste de Tukey. 
XAVIER, J. F.; AZEVEDO, C. A. V.; BeltrÃO, N. E. M.; ANDRADE, A. R. S.; LiMA, V. L. A. Crescimento da mamoneira sob diferentes tipos de águas residuárias e níveis de água no solo. Ambi-Agua, Taubaté, v. 4, n. 3, p. 196-210, 2009. (doi:10.4136/ambi-agua.112)

Tabela 10. Valores médios dos dados de altura da planta (AP), diâmetro caulinar (DC) e área foliar total (AFT) da mamoneira, submetida aos diferentes níveis de água disponível no solo.

\begin{tabular}{|c|c|c|c|}
\hline \multirow{2}{*}{ Níveis de água no solo } & \multicolumn{3}{|c|}{ Variáveis de crescimento } \\
\hline & $\mathbf{A P}(\mathbf{c m})$ & DC (cm) & $\operatorname{AFT}\left(\mathrm{cm}^{2}\right)$ \\
\hline \multicolumn{4}{|c|}{15 Dias Após Semeadura } \\
\hline $\mathrm{N}_{1}=100 \%$ & $11,42 \mathrm{a}$ & 0,053 a & 121,29 a \\
\hline $\mathrm{N}_{2}=80 \%$ & $10,15 \mathrm{a}$ & $0,054 \mathrm{a}$ & 139,99 a \\
\hline $\mathrm{N}_{3}=70 \%$ & $10,67 \mathrm{a}$ & 0,053 a & 152,16 a \\
\hline \multicolumn{4}{|c|}{45 Dias Após Semeadura } \\
\hline $\mathrm{N}_{1}=100 \%$ & 21,67 b & $8,08 \mathrm{~b}$ & $3,016,65$ c \\
\hline $\mathrm{N}_{2}=80 \%$ & $19,07 \mathrm{~b}$ & $6,98 \mathrm{~b}$ & $1,796,18 \mathrm{~b}$ \\
\hline $\mathrm{N}_{3}=70 \%$ & $15,94 \mathrm{a}$ & 4,29 a & 850,63 a \\
\hline \multicolumn{4}{|c|}{60 Dias Após Semeadura } \\
\hline $\mathrm{N}_{1}=100 \%$ & 28,50 c & $12,23 \mathrm{c}$ & $3366,58 \mathrm{c}$ \\
\hline $\mathrm{N}_{2}=80 \%$ & $22,95 \mathrm{~b}$ & $8,93 \mathrm{~b}$ & $21,74 \mathrm{~b}$ \\
\hline $\mathrm{N}_{3}=70 \%$ & 18,08 a & 3,88 a & 947,05 \\
\hline \multicolumn{4}{|c|}{75 Dias Após Semeadura } \\
\hline $\mathrm{N}_{1}=100 \%$ & 30,58 c & $11,86 \mathrm{~b}$ & $3,649,07$ c \\
\hline $\mathrm{N}_{2}=80 \%$ & $25,42 \mathrm{~b}$ & $9,66 \mathrm{ab}$ & $2,406,18 b$ \\
\hline $\mathrm{N}_{3}=70 \%$ & 19,58 a & 6,167 a & $1,140,28$ a \\
\hline \multicolumn{4}{|c|}{120 Dias Após Semeadura } \\
\hline $\mathrm{N}_{1}=100 \%$ & $39,96 \mathrm{c}$ & $14,85 \mathrm{c}$ & 3,746,77 c \\
\hline $\mathrm{N}_{2}=80 \%$ & $32,29 \mathrm{~b}$ & $12,57 \mathrm{~b}$ & $2,845,19 \mathrm{~b}$ \\
\hline $\mathrm{N}_{3}=70 \%$ & 25,83 a & 8,87 a & $1,777,92$ a \\
\hline \multicolumn{4}{|c|}{135 Dias Após Semeadura } \\
\hline $\mathrm{N}_{1}=100 \%$ & $36,50 \mathrm{~b}$ & $17,67 \mathrm{~b}$ & $3,314,98 \mathrm{~b}$ \\
\hline $\mathrm{N}_{2}=80 \%$ & $32,08 \mathrm{ab}$ & $10,98 \mathrm{ab}$ & $2,473,77$ a \\
\hline $\mathrm{N}_{3}=70 \%$ & 26,04 a & 8,04 a & $1,814,84 \mathrm{a}$ \\
\hline
\end{tabular}

Nota: As médias seguidas da mesma letra na coluna não diferem estatisticamente ao nível de 1 e $5 \%$ de probabilidade no Teste de Tukey.

Observa-se por meio da Tabela 9, que as variáveis AP, DC e AFT apresentaram melhores resultados quando submetidas à água residuária $\mathrm{A}_{2}$ (COTEMINAS); esse fato pode ser atribuído, provavelmente, ao conteúdo de nutrientes minerais, principalmente nitrogênio, fósforo e micronutrientes, existente neste tipo de água residuária, como também ao seu elevado teor de matéria orgânica (Tabelas 1 a 3). Com relação aos diferentes níveis de água disponível no solo os melhores resultados foram observados para todas as três variáveis de crescimento da mamoneira, quando submetida ao tratamento $\mathrm{N}_{1}=100 \%$ (Tabela 10).

Utilizou-se a regressão para analisar tendências e comportamento dos valores médios de AP, DC e AFT da mamoneira para o período de 135 DAS, na presença dos diferentes níveis de água disponível do solo. Conforme a Figura 2, verifica-se que todos os valores médios das variáveis relacionadas com o crescimento apresentaram altos coeficientes de determinação $\left(\mathrm{R}^{2}\right)$ com ajuste da equação de forma polinomial de segundo grau para todas as variáveis de crescimento estudadas, apresentando tendência crescente e decrescente. 

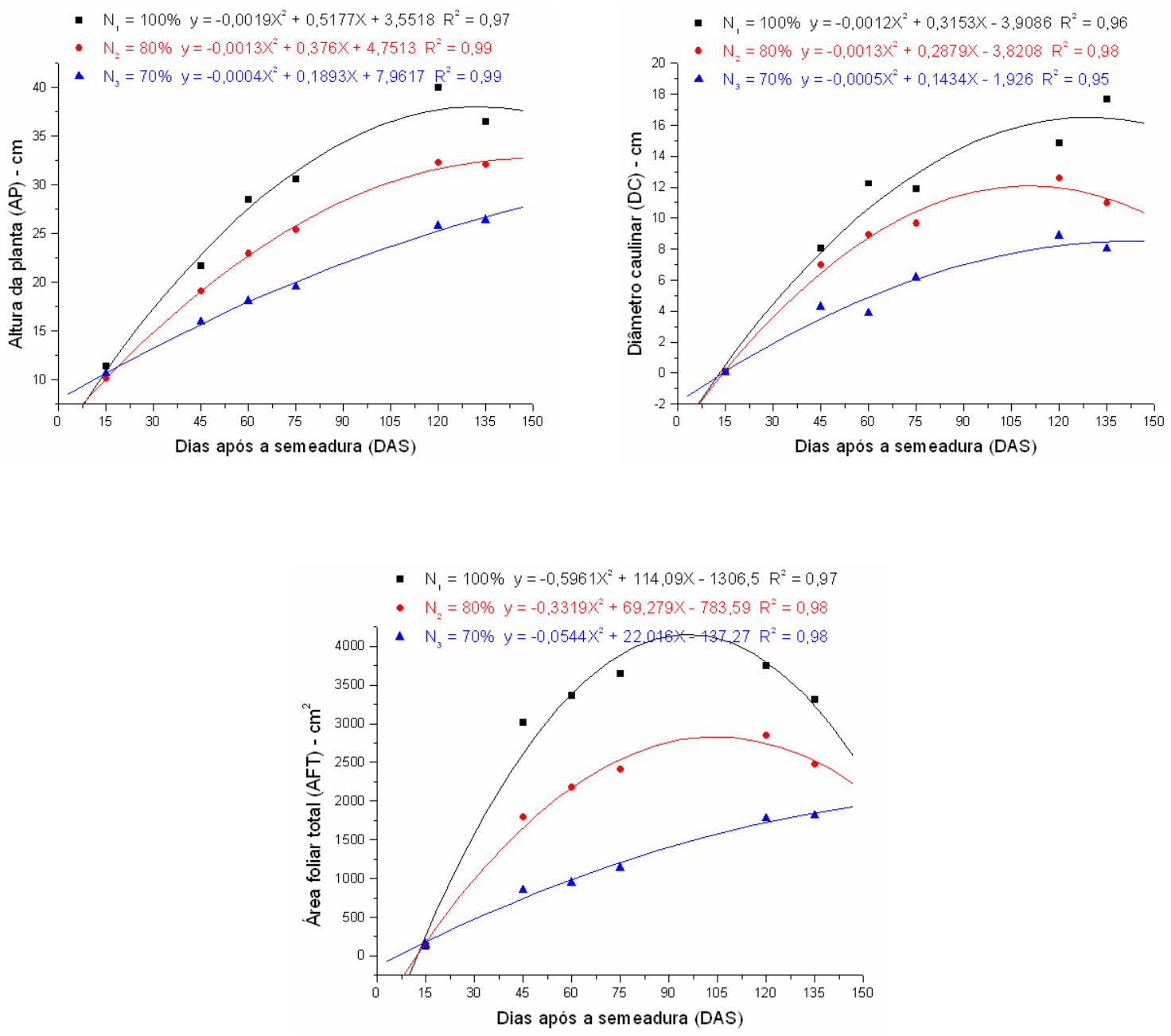

Figura 2. Valores médios dos dados altura da planta (AP), diâmetro caulinar (DC) e área foliar total (AFT) da mamoneira com indicação dos ajustes de regressão para cada nível de água disponível do solo (N).

Na Tabela 11, ao comparar-se os tratamentos tipos de água (A) dentro de cada nível de água disponível do solo $(\mathrm{N})$ na fase final do ciclo da cultura da mamona (135 DAS), observou-se que os melhores resultados ocorreram na interação $A_{2}$ (COTEMINAS) $\mathrm{x} \mathrm{N}_{1}$ (100\%) em todas as variáveis relacionadas com o crescimento da planta, altura de planta (AP), diâmetro do caulinar (DC) e área foliar total (AFT), e os menores valores médios de AP e DC ocorreram quando submetidos ao tratamento $\mathrm{A}_{4}$ (água de abastecimento) x $\mathrm{N}_{1}(100 \%)$, com exceção da AFT foi observado no tratamento $\mathrm{A}_{4}$ (água de abastecimento) x $\mathrm{N}_{2}(80 \%)$. 
XAVIER, J. F.; AZEVEDO, C. A. V.; BeltrÃO, N. E. M.; ANDRADE, A. R. S.; LiMA, V. L. A. Crescimento da mamoneira sob diferentes tipos de águas residuárias e níveis de água no solo. Ambi-Agua, Taubaté, v. 4, n. 3, p. 196-210, 2009. (doi:10.4136/ambi-agua.112)

Verifica-se, na Figura 3, que os maiores valores médios das interações entre os níveis dos fatores analisados (A x N) e as testemunhas para as variáveis AP, DC e AFT em função dos dias após a semeadura (DAS), ocorreram entre 75 e 120 DAS. Observa-se na Figura 3A que as plantas que exibiram maiores valores médios de AP foram aquelas irrigadas com água do tipo $\mathrm{A}_{2}$ e com os níveis de água disponível do solo $\mathrm{N}_{1}(100 \%)$ e $\mathrm{N}_{2}(80 \%)$, ou seja, as interações $\mathrm{A}_{2}$ (COTEMINAS) x $\mathrm{N}_{1}$ e $\mathrm{A}_{2}$ (COTEMINAS) x $\mathrm{N}_{2}(80 \%)$. Nota-se que em geral a mamoneira quando submetida às interações $A_{4} \times N_{1}(100 \%), A_{4} \times N_{2}(80 \%)$ e $A_{4} \times N_{3}(70 \%)$ os valores médios das características de crescimento da planta AP, foram menores quando se irrigou com água de abastecimento e fertilizante na fundação NPK (as três testemunhas).

Tabela 11. Valores médios das interações significativas da análise de variância referente à altura da planta, diâmetro caulinar (DC) e área foliar total (AFT) da mamoneira, submetida a diferentes tipos de água (A) e níveis de água disponível do solo (N) ao final de 135 DAS.

\begin{tabular}{|c|c|c|c|}
\hline Tipos de águas (A) & \multicolumn{3}{|c|}{ Níveis de água disponível no solo } \\
\hline \multirow{6}{*}{$\begin{array}{l}\mathrm{A}_{1}=\text { IPELSA } \\
\mathrm{A}_{2}=\text { COTEMINAS } \\
\mathrm{A}_{3}=\text { LEBOM } \\
\mathrm{A}_{4}=\text { ÁGUA DE } \\
\text { ABASTECIMENTO }\end{array}$} & \multicolumn{3}{|c|}{ Altura da planta (AP) $-\mathrm{cm}$} \\
\hline & 30,66 a $\mathrm{A}$ & 13,50 a $\mathrm{A}$ & 17,66 a $\mathrm{A}$ \\
\hline & 49,33 b A & $48,00 \mathrm{~b} A$ & 39,33 b A \\
\hline & $49.00 \mathrm{~b} \mathrm{~B}$ & $43.33 \mathrm{~b} \mathrm{AB}$ & $31,33 \mathrm{ab} \mathrm{A}$ \\
\hline & 17.00 a $\mathrm{A}$ & 13,50 a $\mathrm{A}$ & 15,83 a $\mathrm{A}$ \\
\hline & \multicolumn{3}{|c|}{ Diâmetro caulinar (DC) - mm } \\
\hline \multirow{5}{*}{$\begin{array}{l}\mathrm{A}_{1}=\text { IPELSA } \\
\mathrm{A}_{2}=\text { COTEMINAS } \\
\mathrm{A}_{3}=\text { LEBOM } \\
\mathrm{A}_{4}=\text { ÁGUA DE } \\
\text { ABASTECIMENTO }\end{array}$} & 13,00 ab A & $8,30 \mathrm{ab} A$ & 0,83 а $\mathrm{A}$ \\
\hline & 25,66 с В & 20,50 с A & 16,33 b A \\
\hline & 20,33 b A & $18,00 \mathrm{~b} \mathrm{~A}$ & $14,33 \mathrm{ab} A$ \\
\hline & 0,70 a $\mathrm{A}$ & 0,63 a $\mathrm{A}$ & 0,66 а $\mathrm{A}$ \\
\hline & \multicolumn{3}{|c|}{ Área foliar total (AFT) $-\mathrm{cm}^{2}$} \\
\hline $\mathrm{A}_{1}=$ IPELSA & $1.189,16$ a $\mathrm{A}$ & 760,71 а A & 318,24 a A \\
\hline $\mathrm{A}_{2}=$ COTEMINAS & $8.787,12$ с В & $7.079,35$ с В & $4.446,93$ с A \\
\hline $\mathrm{A}_{3}=\mathrm{LEBOM}$ & 4.135,64 a A & $3.209,95$ b AB & $2.357,61 \mathrm{~b} \mathrm{~A}$ \\
\hline $\mathrm{A}_{4}=$ ÁGUA DE & 148,02 a $\mathrm{A}$ & 145,08 a A & 136,61 a A \\
\hline ABASTECIMENTO & & & \\
\hline
\end{tabular}

Nota: Médias seguidas da mesma letra, minúscula na linha (dentro dos diferentes tipos de água) e maiúscula na coluna (dentro dos níveis de água no solo), não diferem estatisticamente entre si, pelo teste de Tukey a 1 e $5 \%$ de probabilidade. 
XAVIER, J. F.; AZEVEDO, C. A. V.; BeltrÃO, N. E. M.; ANDRADE, A. R. S.; LiMA, V. L. A. Crescimento da mamoneira sob diferentes tipos de águas residuárias e níveis de água no solo. Ambi-Agua, Taubaté, v. 4, n. 3, p. 196-210, 2009. (doi:10.4136/ambi-agua.112)

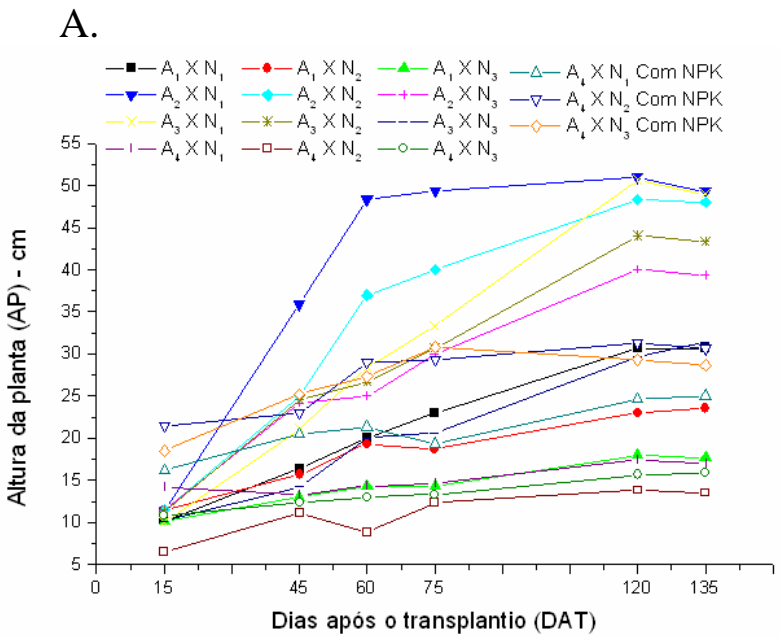

B.

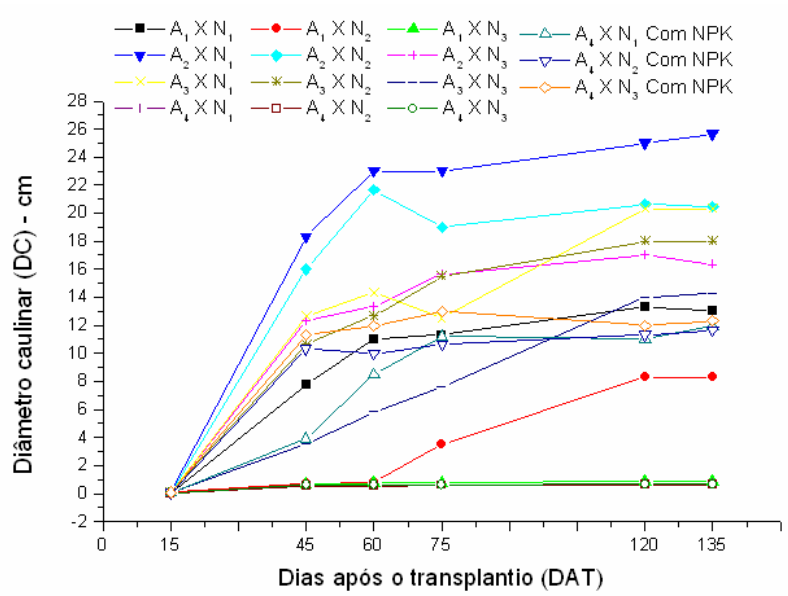

C.

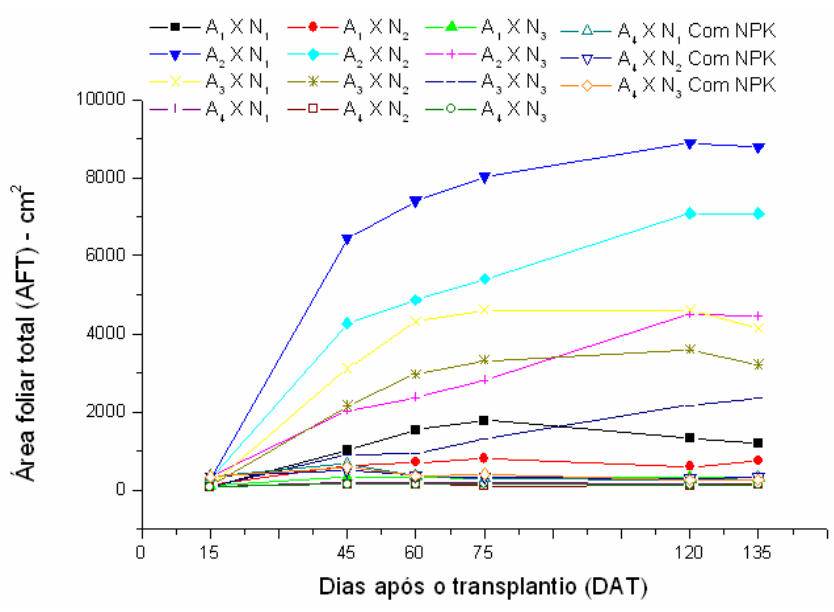

Figura 3. Valores médios da altura da planta (A), do diâmetro caulinar (B) e da área foliar total (C) da mamoneira, para as interações entre os níveis dos fatores analisados $(\mathrm{A} \times \mathrm{N})$ versus as testemunhas em função dos dias após a semeadora. 
Observa-se que as plantas que exibiram maiores valores médios de DC (Figura 3B) e AFT (Figura 3C) foram aquelas irrigadas com água do tipo $A_{2}$ (COTEMINAS) e com os níveis de água disponível do solo $\mathrm{N}_{1}$, e, em geral, observa-se que a mamoneira quando submetida às interações $A_{4} \times N_{1}, A_{4} \times N_{2}$ e $A_{4} \times N_{3}$ os valores médios das variáveis $D C$ e AFT foram menores nas três testemunhas.

No geral, constata-se que a mamoneira, cultivar BRS Nordestina, respondeu bem à irrigação com água residuária tratada, em especial da indústria COTEMINAS, principalmente quando associada ao nível de $100 \%$ da água disponível no solo, a mais rica e equilibrada em nutrientes minerais entre as testadas (IPELSA e LEBOM), mesmo considerando o tratamento com água de abastecimento sendo adubado com NPK. Com o uso de somente água de abastecimento, as plantas da mamoneira nem se quer iniciaram a floração, e ficaram raquíticas e desnutridas, com sintomas de deficiências de diversos nutrientes, em especial, nitrogênio e fósforo.

\section{CONCLUSÕES}

Para todas as variáveis de crescimento (altura de plantas, diâmetro caulinar e área foliar total em várias épocas), houve interações significativas entre os fatores estudados, denotando a interdependência entre eles, o que foi refletido no crescimento das plantas.

A mamoneira, cultivar BRS Nordestina, respondeu bem à irrigação com água residuária tratada, em especial da indústria COTEMINAS, principalmente, quando associada ao nível de $100 \%$ da água disponível no solo.

\section{REFERÊNCIAS}

AMORIM NETO, M. da S.; BELTRÃO, N. E. de M. Zoneamento do algodão herbáceo no nordeste. In: BELTRÃO, N. E. de M. (org.). O agronegócio do Algodão no Brasil. Brasília: Embrapa Comunicação para transferência de Tecnologia, 1999.v.1 (2v.),p.213229.

ARAÚJO, A. E. de; AMORIM NETO, M. da S.; BELTRÃO, N. E. de M. Municípios aptos e épocas de plantio para o cultivo da mamoneira no Estado da Paraíba. Revista de Oleaginosas e Fibras, Campina Grande, v. 4, n.2, p.103-110, 2000.

AZEVEDO, D. M. P.; BELTRÃO, N. E. de M.; NÓBREGA, L. B.; VIEIRA, D. J. O agronegócio da Mamona no Brasil. Brasília: Embrapa Informação Tecnologia, 2001.

BELTRÃO, N. E. DE M.; SILVA, L. C.; VASCONCELOS, O. L.; AZEVEDO, D. M. P.; VIEIRA, D. J. O agronegócio da Mamona no Brasil. Brasília: Embrapa Comunicação para Transferência de Tecnologia, 2001. cap. 2, Fitologia. p.37-59.

BUSSAB, W. O.; MORETTIN, P. A. Estatística básica. 5. ed. ed. São Paulo: Saraiva, 2003. 526p.

COELHO, M. A.; SONCIN, N. B. Geografia do Brasil. São Paulo: Moderna, 1982. 368p.

EMPRESA BRASILEIRA DE PESQUISA AGROPECUÁRIA - EMBRAPA. Centro Nacional de Pesquisa dos Solos. Manual de métodos de análise de solo. 2. ed. Rio de Janeiro: EMBRAPA - CNPS, 1997. 212p.

INSTITUTO BRASILEIRO DE GEOGRAFIA E ESTATÍSTICA - IBGE. Anuário Estatístico do Brasil. Rio de Janeiro: IBGE, 1991. 
XAVIER, J. F.; AZEVEDO, C. A. V.; BeltrÃO, N. E. M.; ANDRADE, A. R. S.; LIMA, V. L. A. Crescimento da mamoneira sob diferentes tipos de águas residuárias e níveis de água no solo. Ambi-Agua, Taubaté, v. 4, n. 3, p. 196-210, 2009. (doi:10.4136/ambi-agua.112)

INSTITUTO BRASILEIRO DE GEOGRAFIA E ESTATÍSTICA - IBGE. Anuário Estatístico do Brasil. Rio de Janeiro: IBGE, 2000.

MANCUSO, P. S; SANTOS, H. F. Reúso de água. Barueri: Manole, 2003.

SANTOS, J. W. dos; AlMEIDA, A. C.; BELTRÃO, N. E. de M.; CAVALCANTE, F. B. Estatística Experimental Aplicada. 2. ed. revisada e ampliada. Campina Grande: Embrapa Algodão/ Universidade Federal de Campina Grande, 2008.

SILVA, S. A.; OLIVEIRA R. de. Manual de Análises Físico-químicas de Água de Abastecimento e Residuárias. Campina Grande: UFCG, 2001. 266p.

SOUSA, J. T.; LEITE, V. D. Tratamento e utilização de esgotos domésticos na agricultura. Campina Grande: EDUEP, 2003. 103p

STUDART, T.; CAMPOS, N. Gestão das Águas: princípios e práticas. 2. ed. Porto Alegre: ABRH, 2001.

VON SPERLING, M. Noções de qualidade de água. In: Princípios do tratamento biológico de águas residuárias. Belo Horizonte: UFMG, 1996. 187p. 